Courrier du Centre international Blaise Pascal

$9 \mid 1987$

Varia

\title{
Pascal en Sorbonne (1986-1987)
}

Philippe Sellier

\section{OpenEdition}

Journals

Édition électronique

URL : http://journals.openedition.org/ccibp/452

DOI : $10.4000 /$ ccibp.452

ISSN : 2493-7460

\section{Éditeur}

Centre international Blaise Pascal

\section{Édition imprimée}

Date de publication : 4 septembre 1987

Pagination : 26-27

ISSN : 0249-6674

\section{Référence électronique}

Philippe Sellier, «Pascal en Sorbonne (1986-1987) », Courrier du Centre international Blaise Pascal [En ligne], 9 | 1987, mis en ligne le 07 décembre 2015, consulté le 23 avril 2019. URL : http:// journals.openedition.org/ccibp/452 ; DOI : 10.4000/ccibp.452

Ce document a été généré automatiquement le 23 avril 2019.

Centre international Blaise Pascal 


\title{
Pascal en Sorbonne (1986-1987)
}

\author{
Philippe Sellier
}

1 Le mois de mars 1985 avait vu se succéder en Sorbonne deux soutenances marquantes de thèses de doctorat d'État: Les Pensées de Pascal. 1670-1734 d'Antony McKenna et L'Argumentation chez Pascal de Dominique Descotes. À huit jours d'intervalle (9 et 16 mars). Cette vitalité des études pascaliennes avait conduit l'un des membres du jury, éminent spécialiste des formes brèves, à une sentence flatteuse pour les pascaliens : «Ce n'est plus une chapelle, c'est une cathédrale ».

2 L'année 1986-1987 n'a pas été moins brillante, puisque se sont succédé à Paris-Sorbonne quatre soutenances de doctorat de III $^{\mathrm{e}}$ cycle (la dernière consistait en fait en un doctorat "nouvelle formule» comparable aux meilleurs $\mathrm{III}^{\mathrm{e}}$ cycles). Ces travaux étaient d'excellente qualité, ce qui a valu à chacun d'eux la mention la plus élevée.

3 Tout commença avec $M^{l l e}$ Ran-E. Hong, Coréenne du Sud et benjamine des études pascaliennes. Le 14 janvier 1987, elle présenta une thèse intitulée Le Dialogue chez Pascal : magnifique sujet, dont on s'étonne qu'il ait été longtemps si peu étudié. Avec une rare élégance d'écriture et une ample culture philosophique (de Platon à Kierkegaard et à Bergson), $\mathrm{M}^{\text {lle }}$ Hong s'est livrée à une réflexion approfondie sur les problèmes généraux de la forme dialoguée chez Pascal : lien à certaines figures de rhétorique, connivence avec l'inachèvement, possibilités et limites de cette forme, etc. Le jury n'a regretté qu'un certain évitement des analyses techniques, à ras de texte, contrepartie de l'altitude à laquelle $\mathrm{M}^{\text {lle }}$ Hong a choisi de se placer.

4 Puis vint M. Laurent Thirouin, le 6 février 1987, avec un livre éblouissant Le Modèle du jeu chez Pascal (Le Hasard et les Règles), fragment de sa future thèse de doctorat d'État, Le Jeu chez Pascal. Trois critères caractérisent le jeu, selon L. Thirouin : l'insignifiance des objets qu'il se propose, l'arbitraire des règles mises en place, le plaisir que procure le processus. Cette détermination pose divers problèmes : peut-on parler d'un jeu de Dieu ? N'existe-t-il pas de jeu sans règles ? Des pages très fortes mettent en pleine lumière l'importance de la notion de hasard. Suit une analyse brillante et convaincante sur la règle du jeu appliquée à l'espace social : le travail des hommes politiques consiste à présenter comme des effets de la raison ce qui n'est que règles ludiques. De là chez Pascal une pratique humoristique 
des gestes sociaux. Le livre s'achève sur une mise au point minutieuse et exigeante à propos du fragment «Infini rien ». Ces quatre-vingt pages incisives viennent déjà de provoquer deux élèves en fin de formation à l'École des Hautes Études Commerciales (H.E.C.) à choisir comme Mémoire de théorie financière l'analyse mathématique du Pari à la lumière des théories modernes de la prise de décision. De là ce titre prometteur: Le Discours de la machine, ou Pascal spéculateur.

Revenons à la Corée du Sud. Le 13 mai 1987, $\mathrm{M}^{\text {lle }}$ Myung-Ai Cho présenta sa thèse L'Homme et Dieu chez Pascal et Lao-tseu. Au jury siégeait le R. Père Houang, co-auteur d'une des traductions françaises du Tao-toking. Le genre risqué du parallèle, loin de se révéler ici peu fécond, aboutit à des rapprochements inattendus, qui manifestent entre deux cultures et deux ensembles de textes apparemment éloignés l'existence d'une sorte de fond commun anthropologique : pensée des contraires, de l'entre-deux, du point de vue; règne de la dialectique et du paradoxe. Bien sûr, on ne saurait masquer ce qui sépare le Dieu personnel du christianisme et l'union à un Ordre caché de l'univers en perpétuel devenir ; ou la liberté chrétienne et une simple soumission à l'Ordre du monde. Mais $\mathrm{M}^{\text {lle }}$ Cho esquisse brillamment l'étude de l'importance de la rythmique dans l'imagination et la pensée pascaliennes.

6 Enfin, le 5 septembre 1987, dans une Sorbonne encore quasi déserte, se tint une ultime soutenance, celle de M. Pierre Force de l'université Columbia (New York), sur Le Sens d'un auteur. Étude du problème herméneutique chez Pascal. Doté d'une vaste culture (de la Bible à la philosophie et aux mathématiques), l'auteur met en évidence de façon saisissante le rôle central de l'herméneutique chez Pascal: celle-ci traite les textes de la théologie moderne (contre l'herméneutique jésuite), l'œuvre augustinienne, les Écritures... M. Force souligne avec justesse l'intérêt de Pascal pour la cryptographie (le «chiffre»). La perspective adoptée permet d'embrasser non seulement les Provinciales et les Pensées, mais aussi les Écrits sur la grâce et même certaines œuvres scientifiques. Même si l'étude majore peut-être un peu la rigueur géométrique de Pascal en toutes ses démarches, elle fait apparaître l'œuvre comme encore plus rigoureuse et plus cohérente qu'on ne croyait.

Si nous consentons à nous éloigner un peu de la Sorbonne, il faut mentionner aussi la thèse de doctorat d'État de M. Fernand Reusser, soutenue à Rouen le 11 juin 1986, Fidéisme et rationalisme. Pascal et ses contemporains. Moins soucieux d'innover que de constituer sa synthèse personnelle sur les rapports entre foi et raison à partir de Pascal, M. Reusser atteste la fascination persistante exercée par les Pensées.

8 Enfin cette riche année 1986-1987 fut couronnée par la Journée PASCAL de l'Association Internationale des Études Françaises, au Collège de France, le 23 juillet 1987. $\mathrm{M}^{\text {lle }}$ Hong et M. Thirouin y parlèrent, en compagnie de leurs aînés, MM. Descotes et McKenna. Les communications paraitront en mai-juin 1988, dans les Cahiers de l'Association (CAIEF). Il importe de souligner que cette Journée fut présidée par M. Jean Mesnard, qui avait été le Directeur de toutes les thèses soutenues en Sorbonne et citées précédemment.

M. Mesnard dirige également deux thèses dont l'achèvement est tout proche : le doctorat d'État de M. Pol Ernst sur les «avant-textes » des Pensées (couches rédactionnelles, reconstitution des grandes feuilles d'origine, classement et datation des papiers, etc.); et l'édition du Recueil de choses diverses (B.N. ms fr. 4333), préparée par M. Jean Lesaulnier. La Corée du Sud n'a pas dit non plus son dernier mot.

10 La plupart des pascaliens ici présentés se retrouveront aux colloques de Tokyo et du Kansai (24 septembre-9 octobre 1988). Mais comme tous ceux qui travaillent sur Pascal ne 
se rencontreront malheureusement pas à cette occasion, le C.I.B.P. lance un appel pour que des bilans analogues à celui-ci soient établis dans les différents pays où nos études sont particulièrement actives. En les publiant, le Courrier jouera pleinement son rôle de support d'information et de documentation, au service de tous.

\section{Errata}

11 La thèse de troisième cycle de $\mathrm{M}^{\mathrm{lle}}$ Ran-E Hong, Le Dialogue chez Pascal. De la forme dialoguée à la dialectique, a été soutenue en 1987. Elle comporte 232 pages. Au jury figurait M. AlainPierre Cahné et non le père Houang. $\mathrm{M}^{\text {lle }}$ Hong n'appartenait pas alors à l'Université de Séoul.

\section{AUTEUR}

PHILIPPE SELLIER

Université Paris V 